\title{
Autologous Blood Injection Works for Recalcitrant Lateral Epicondylitis
}

\author{
Bora Bostan ${ }^{1}$, Orhan Balta ${ }^{1}$, Murat Aşç1 ${ }^{1}$, Kürşad Aytekin ${ }^{2}$, Enes Eser $^{3}$ \\ ${ }^{1}$ Department of Orthopaedics and Traumatology, Gaziosmanpaşa University School of Medicine, Tokat, Turkey \\ ${ }^{2}$ Department of Orthopaedics and Traumatology, Giresun University School of Medicine, Giresun, Turkey \\ ${ }^{3}$ Department of Orthopaedics and Traumatology, Turhal State Hospital, Tokat, Turkey
}

Background: Recalcitrant lateral epicondylitis may be a disabling condition. Treatment of this condition is still controversial.

Aims: In the present prospective study, we evaluated the long-term results of autologous blood injection for the treatment of recalcitrant lateral epicondylitis.

Study Design: Prospective clinical study.

Methods: A total of 42 elbows of 40 consecutive patients ( 28 female, 12 male) were enrolled in this prospective study. Seven patients left the study (3 patients moved to another city, 1 patient died in the second week due to a heart condition, 1 patient quit the study because of the resolution of pain in the fourth week and 2 patients did not agree to the second injection). Thirteen patients were lost to third year follow-up.
Therefore, a total of 21 elbows of 20 patients with 3 years of follow-up were included in this study. The mean age of the patients was 47.25 years (range, 2068 years).

Results: Visual analogue scale (VAS), Nirschl score and grip strength were significantly improved after injections when compared to before treatment. The best improvement in terms of grip strength, Nirschl score and VAS score was detected at the one year follow-up. The improvement in Nirschl and VAS score sustained until the third year.

Conclusion: We suggest that autologous blood injection for the treatment of recalcitrant lateral epicondylitis is an effective, safe and successful procedure in the long-term. Keywords: Autologous blood, tennis elbow, lateral epicondylitis
Lateral epicondylitis (tennis elbow) is a frequent condition that is characterized by activity-related pain, focal tenderness and decreased strength in the lateral elbow (1). It is an overuse tendinopathy of the wrist extensors at the humeral attachment (2). The precise treatment of this condition is yet to be elucidated because the pathophysiology of the disease is not clearly understood (2-7). Various treatments such as physical therapy, non-steroidal anti-inflammatory drugs, local corticosteroid injections, arthroscopic surgery, topical nitric oxide and platelet rich plasma injection have all been used with different efficacy and cost effectiveness (2-7). Although there are many treatment choices, some patients may not respond to these treatments, and are referred to as recalcitrant cases. The two most commonly used treatments, non-steroidal anti-inflammatory drugs and local corticosteroids, have no beneficial effects on intermediate and long-term follow-up $(6,7)$. This may be attributed to the absence of inflammation in the pathogenesis of disease.

Although there has been a lot of research on the treatment of lateral epicondylitis, especially with platelet-rich plasma and autologous blood injection, there is a lack of studies investigating the treatment of recalcitrant cases with autologous blood injection. In addition, the existing studies do not reveal any intermediate or long-term results (8-14). Recalcitrant cases can be treated with surgical procedures. Unfortunately, surgery, particularly open surgery, has a prolonged healing time; approximately $10 \%$ of the patients may remain symptomatic after surgery (5). 
In the present prospective study, we evaluated the long-term results of autologous blood injection for the treatment of recalcitrant lateral epicondylitis.

\section{MATERIALS AND METHODS}

A total of 42 elbows of 40 consecutive patients ( 28 female, 12 male) were enrolled in this prospective study. However, seven patients left the study for the following reasons: 3 patients moved to other cities, 1 died in the second week (due to a heart condition), 1 was completely relieved of pain in the fourth week and did not return and 2 left because of persisting symptoms and refusal of the second injection after the fourth week. Also, 13 patients were lost to the third year follow-up. Therefore, a total of 21 elbows of 20 patients with 3 years follow-up were included in this study. The mean age of the patients was 47.25 years (range: $20-68$ years).

The trial was approved by the Ethics Committee of the School of Medicine. Informed consent was obtained from the patients. Pain over the lateral epicondyle with direct palpation and with resisted wrist extension was defined as lateral epicondylitis. To rule out other periarticular conditions such as loose bodies, tumor, or arthrosis, AP and lateral roentgenography of the elbow was obtained prior to the injections. Once the diagnosis was made, patients were asked to rate their pain on a 0-100 visual analogue scale before treatment (VAS BT) ( 0 refers to no pain, and 100 the worst pain) before injection. Functional scoring was performed with the modified Nirschl score before treatment (NS BT) (13), with phase 1 representing the best functional score and phase 7 representing the worst (Table 1).

Grip strength before treatment (GS BT) was measured with a dynamometer in kilograms (Jamar, Lafayette Instruments; Lafayette, USA).

All patients had failed previous nonsurgical treatments such as nonsteroidal anti-inflammatory drug (NSAID), physical therapy, bandage, and local corticosteroid injection or a combination of these treatments. Inclusion criteria were as follows: a VAS score greater than 40 , which indicates serious pain, duration of the symptoms greater than 3 months, and

TABLE 1. Nirschl staging of lateral epicondylitis

\begin{tabular}{ll}
\hline Phase 1 & Mild pain with exercise, resolves within 24 hour \\
Phase 2 & Pain after exercise, exceeds 24 hour \\
Phase 3 & Pain with exercise and does not alter activity \\
Phase 4 & Pain with exercise and alters activity \\
Phase 5 & Pain with heavy activities of daily living \\
Phase 6 & Pain with light activities of daily living and intermittent pain at rest \\
Phase 7 & Constant pain at rest, disrupts sleep \\
\hline
\end{tabular}

pain that is recalcitrant to other treatments. Exclusion criteria were pregnancy (hormonal and psychological changes may interfere with the results), carpal tunnel syndrome, cervical radiculopathy, elbow arthritis, loose bodies, elbow surgery and a VAS score less than 40 , history of recent trauma, and systemic conditions such as diabetes, rheumatoid arthritis, hepatitis etc.

The standard injection protocol used for all patients consisted of $0.5 \mathrm{~mL}$ bupivacaine (Marcaine, AstraZeneca; Sweden, UK) injection to the skin, subcutaneous tissues and area of the maximum tenderness. Subsequently, $3 \mathrm{~mL}$ of autologous blood drawn from the contralateral arm was injected into the tendon at the area of maximum tenderness after the dry needling technique, which is defined as making multiple punctures of the area with maximum tenderness from one skin entry point (15). Initially, one injection per patient was planned, but a second injection was made in four patients at the end of the fourth week because of persisting symptoms. We did not use any limitations or physical therapy and splints after the procedure. Paracetamol (Minoset $500 \mathrm{mg}$, Bayer; İstanbul, Turkey) was prescribed in case of pain. The patients were examined at 2, 4 and 6 weeks, 6 months, 1 year and 3 years in terms of VAS score (VAS PT). Nirschl score was measured at 4 and 6 weeks, 6 months, 1 year and 3 years (NS PT). Grip strength was measured at 2, 4 and 6 weeks, 6 months, and 1 year (GS PT). Since the third year questionnaire for 5 patients was performed by phone, grip strength of these patients was not measured; therefore, grip strength in the third year was not taken into consideration.

\section{Statistical analysis}

One-Sample Kolmogorov-Smirnov test was used to evaluate whether the distribution of continuous variables was normal. Friedman test was used to compare the scores between pre- and post-treatment terms. For multiple comparisons, Wilcoxon Signed Rank Test with Bonferroni Correction was used. Variables were presented as the mean \pm standard deviation. A p value $<0.05$ was considered significant. Analyses were performed using commercial software (IBM Corp. Released 2010. IBM SPSS Statistics for Windows, Version 19.0 Armonk, IBM Corp.; NY, USA).

\section{RESULTS}

The average duration of the symptoms prior to treatment was 8.4 months (range, 3-72 months). Before registration to the current study, the average number of prior treatments was 1.7 (range, 1-7). VAS, Nisrchl score and grip strength were significantly improved after injections when compared to before treatment (Table 2, Figure 1-3) $(\mathrm{p}<0.001)$. The best im- 
TABLE 2. The results of VAS, NS, GS before and after treatment

\begin{tabular}{lcccccccc}
\hline & $\mathrm{BT}$ & 2 weeks & 4 weeks & 6 weeks & 6 months & 1 year & 3 years & $\mathrm{p}$ \\
\hline VAS Score & $81.67 \pm 12.00$ & $46.11 \pm 20.04^{*}$ & $25.55 \pm 17.22^{*}$ & $14.44 \pm 19.39^{*}$ & $3.89 \pm 9.78^{*}$ & $1.67 \pm 7.07^{*}$ & $2.22 \pm 5.48^{*}$ & $<0.001$ \\
NS Score & $5.55 \pm 1.10$ & - & $1.94 \pm 1.21^{* *}$ & $1.00 \pm 0.97^{* *}$ & $0.22 \pm 0.54^{* *}$ & $0.17 \pm 0.51^{* *}$ & $0.17 \pm 0.38^{* *}<0.001$ \\
GS Score & $27.43 \pm 11.84$ & $42.14 \pm 12.62^{* * *}$ & $50.28 \pm 12.90^{* * *}$ & $57.48 \pm 18.43 * * *$ & $61.19 \pm 15.56^{* * *}$ & $69.76 \pm 22.44^{* * *}$ & - & $<0.001$ \\
\hline
\end{tabular}

* Significant Difference Between VAS_BT

**Significant Difference Between NS_BT

***Significant Difference Between GS_BT

Data were presented as Mean \pm Std. Deviation

VAS: visual analogue scale; NS: Nirschl score; GS: grip strength; BT: before treatment

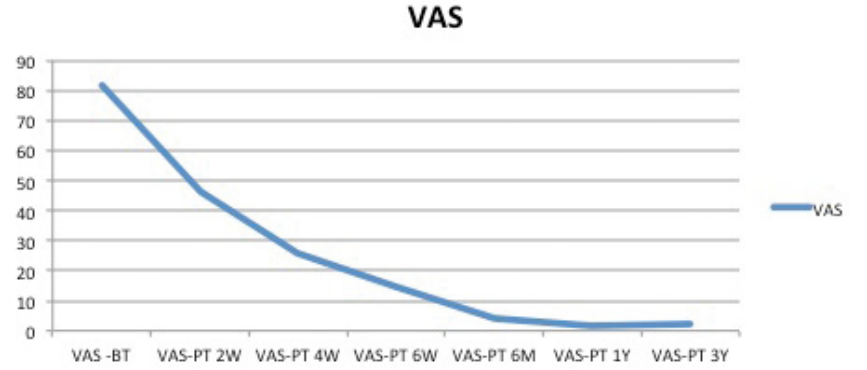

FIG. 1. The results of VAS scores before and after treatment (VAS BT: VAS before treatment; VAS PT: VAS post-treatment)

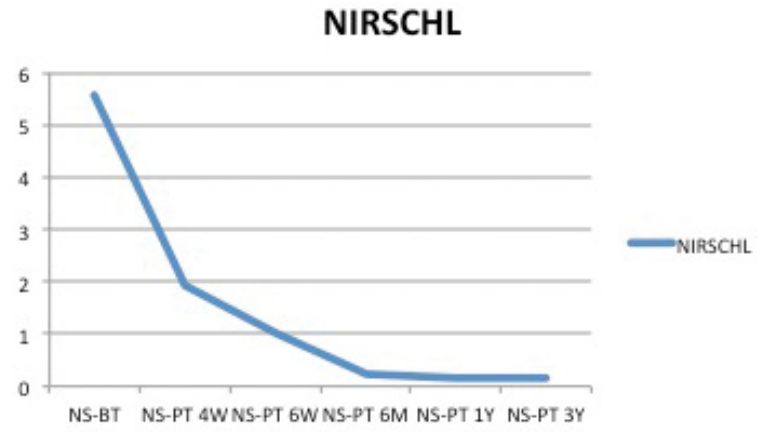

FIG. 2. The results of Nirschl scores before and after treatment (NS BT: Nirschl score before treatment; NS PT: Nirschl score post-treatment)

\section{GRIP STRENGTH (KG)}

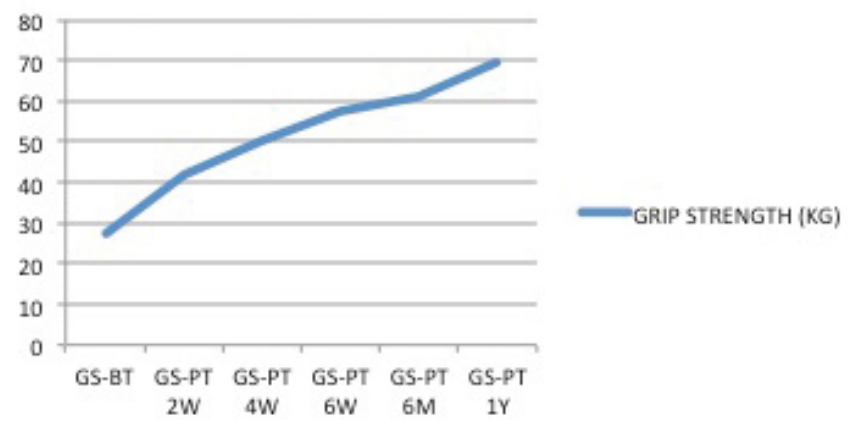

FIG. 3. The results of grip strength before and after treatment (GS BT: grip strength before treatment; GS PT: grip strength post-treatment) provement in terms of grip strength, Nirsch score and VAS score was detected at one year follow-up. The improvement in Nirsch and VAS score was sustained until the third year.

\section{DISCUSSION}

The pathogenesis of tendinopathy is triggered by a high dose cyclic strain that causes oxidative stress and the induction of cartilage genes. These two major pathways lead to apoptosis and a loss of matrix integrity, causing metalloproteinase activity. As a result, a degeneration and regeneration process begins. This process is accompanied by vascular infiltration and nerve regeneration (1). Therefore, the longterm effectiveness of NSAIDs for the treatment of lateral epicondylitis has fallen into question in recent years. In this regard, Edwards and Calandruccio (11) suggested the possible mechanism of action of autologous blood injection to be that chemical modifiers, which are known to be mitomorphogenic and carried in the whole blood, provide the necessary cellular and humoral mediators to induce a healing cascade of degenerated tendinous origin of extensor carpi radialis brevis (11). In a review where fourteen studies were evaluated, the effects of NSAIDs in treating lateral epicondylitis pain were investigated. The median follow-up was 2 weeks (range, 1-12 weeks). The authors suggested that topical and oral NSAIDs have short-term benefits, while oral NSAIDs cause gastrointestinal complications (6). Another review performed by Smidt et al. (7) evaluated thirteen randomized controlled studies regarding the use of local corticosteroid injections in tennis elbow and found that local corticostreoid injections have superior benefit (pain, global improvement and grip strength) in the short-term ( $\leq 6$ weeks) compared to placebo, local anesthetic and conservative treatment. However, this benefit was not sustained in the intermediate (6 weeks- 6 months) or long-term ( $\geq 6$ months). The mechanism of short-term relief following steroid injection is not clear. It may, however, be attributed to bleeding into the degenerated area thorough fenestrations by needling (12). 
A recent study comparing the effectiveness of local corticosteroid and autologous blood injection for lateral epicondylitis revealed that the autologous blood injection group showed a statistically significant decrease in pain compared with corticosteroid injection at the 12-week and six month follow-up (9). Edwards and Calandruccio (11) studied autologous blood injections for refractory lateral epicondylitis; the patients were followed-up for an average of 9.5 months. Twenty-two of the twenty-eight patients were completely free of pain; therefore, they advocated autologous blood injection, which addresses the pathophysiology of the disease (11). Ultrasonographyguided autologous blood injection was found to be beneficial in tendinosis of different locations with a follow-up greater than 10 months. These studies showed that healing could be monitored by ultrasonography by detecting echo-texture, intertstitial tears and neovascularity $(12,14,15)$. Autologous blood injection for lateral epicondylitis can be performed by ultrasonography guidance or alone. The results for both methods are good $(11,15-17)$. However, studies that compare the results of autologous of blood injection for lateral epicondylitis with or without ultrasonography guidance do not exist. In contrast to the literature, Wolf et al. (8) found no additional benefit of autologous blood injection over corticosteroid or saline injection and stated that tendinopathy of extensor carpi radialis brevis origin is self-limited. Since lateral epicondylitis may be self-limited in some patients, we performed the study in a cohort consisting of recalcitrant cases and thus ruled out the possibility of self-limitation.

To the best of our knowledge, five years is the longest follow-up of patients treated with autologous blood injection for lateral epicondylitis with excellent results. In this study, patients wore customized wrist supports for five days after the injection and then commenced a stretching and strengthening program. The authors concluded that autologous blood injection is effective in patients who had no relief with cortisone injection (16). Although the mean follow-up and results of our study were close to the results reported by Massy-Westropp et al. (16), the post-injection follow-up protocol was different. We did not use a brace or stretching and strengthening exercises after the autologous blood injections, which is costeffective and more comfortable. In a study by Thanasas et al. (17), platelet rich plasma and autologous blood injection for chronic tennis elbow were compared and it was found that VAS score improvement was statistically significant in favor of PRP only at 6 weeks. The Liverpool elbow score was not significantly different between groups. They stated that PRP is not cost-effective over autologous blood injection for the treatment of chronic, refractory tennis elbow (17). The treatment of recalcitrant lateral epicondylitis is still a subject of debate. In a study by Mullett et al. (5), thirty patients suffering from recalcitrant lateral epicondylitis were treated with arthroscopic surgery and followed-up for an average of 9 months. A1though they were pleased with the results, $10 \%$ of the patients had pain during daily activities (5). Surgery is an invasive procedure and may not be cost-effective. Since corticosteroid injections do not address the pathophysiology of the disease, the results are not sustained in the long-term. Therefore, the topical application of nitric oxide is another choice. Since these procedures have some disadvantages, such as complications and cost, autologous blood injection can be favored over these procedures with the advantage of cost effectiveness and absence of complications. To the best of our knowledge, this is the first study with a long follow-up to investigate the results of recalcitrant lateral epicondylitis treated with autologous whole blood injection with no additional brace or exercise.

The limitations of this study include the lack of a randomized control group, the approximately 50\% loss to follow-up at 3 years and not measuring third year grip strength in all patients.

In conclusion, we suggest that autologous blood injection with no additional brace or exercise is an effective, safe and successful procedure for the treatment of recalcitrant lateral epicondylitis in the long-term.

Ethics Committee Approval: Ethics committee approval was received for this study from the ethics committee of Gaziosmanpaşa University Ethical Committee, 09-GEKTIP-003.

Informed Consent: Written informed consent was obtained from patients who participated in this study.

Peer-review: Externally peer-reviewed.

Author contributions: Concept - B.B.; Design - B.B.; Supervision - B.B.; Resource - B.B., M.A.; Materials - B.B.; Data Collection and/or Processing - B.B., M.A., O.B., E.E., K.A.; Analysis and/or Interpretation - B.B., M.A., O.B.; Literature Search - B.B., M.A., O.B., E.E., K.A.; Writing - B.B.; Critical Reviews - B.B., M.A., O.B., K.A.

Conflict of Interest: No conflict of interest was declared by the authors.

Financial Disclosure: The authors declared that this study has received no financial support.

\section{REFERENCES}

1. Xu Y, Murrell GA. The basic science of tendinopathy. Clin Orthop Relat Res 2008;466:1528-38. [CrossRef]

2. Mishra A, Pavelko T. Treatment of chronic elbow tendinosis with buffered platelet-rich plasma. Am J Sports Med 2006;34:1774-8. [CrossRef] 
3. Paoloni JA, Appleyard RC, Nelson J, Murrell GA. Topical nitric oxide application in the treatment of chronic extensor tendinosis at the elbow: A randomized, double blinded, placebo controlled clinical trial. Am J Sports Med 2003;31:915-20.

4. Struijs PA, Kerkhoffs GM, Assendelft WJ, Van Dijk CN. Conservative treatment of lateral epicondylitis: Brace versus physical therapy or a combination of both- A randomized clinical trial. Am J Sports Med 2004;32:462-9. [CrossRef]

5. Mullett H, Sprague M, Brown G, Hausman M. Arthroscopic treatment of lateral epicondylitis: Clinical and cadaveric study. Clin Orthop Relat Res 2005;439:123-8. [CrossRef]

6. Green S, Buchbinder R, Barnsley L, Hall S, White M, Smidt N, et al. Non-steroidal anti-inflammatory drugs (NSAID) for treating lateral elbow pain in adults. Cochrane Database Syst Rev 2001;4:1-18. [CrossRef]

7. Smidt N, Assendelft WJ, van der Windt DA, Hay EM, Buchbinder R, Bouter LM. Corticosteroid injections for lateral epicondylitis: a systematic review. Pain 2002;96:23-40. [CrossRef]

8. Wolf JM, Ozer K, Scott F, Gordon MJ, Williams AE. Comparison of autologous blood, corticosteroid, and saline injection in the treatment of lateral epicondylitis: A prospective, randomized, controlled, multicenter study. J Hand Surg Am 2011;36:1269-72. [CrossRef]

9. Dojode CM. A randomized control trial to evaluate the efficacy of autologous blood injection versus local corticosteroid injection for the treatment of lateral epicondylitis. Bone Joint Res 2012;1:192-7. [CrossRef]
10. Jindal N, Gaury Y, Banshiwal RC, Lamoria R, Bachhal V. Comparison of short term results of single injections of autologous blood and steroid injection in tennis elbow: a prospective study. J Orthop Surg Res 2013;8:1-7. [CrossRef]

11. Edwards SG, Calandruccio JH. Autologous blood injection for refractory lateral epicondylitis. J Hand Surg Am 2003;28:272-8. [CrossRef]

12. Connell DA, Ali KE, Ahmad M, Lambert S, Corbett S, Curtis M. Ultrasound-guided autologous blood injection for tennis elbow. Skeletal Radiol 2006;35:371-7. [CrossRef]

13. Nirschl RP. Elbow tendinosis/tennis elbow. Clin Sports Med 1992;11:851-70.

14. James SL, Ali K, Pocock C, Robertson C, Walter J, Bell J, et al. Ultrasound guided dry needling and autologous blood injection for patellar tendinosis. Br J Sports Med 2007;41:518-21. [CrossRef]

15. Suresh SP, Ali KE, Connell DA. Medial epicondylitis: is ultrasound guided autologous blood injection an effective treatment. Br J Sports Med 2006;40:935-9. [CrossRef]

16. Massy-Westropp N, Simmonds S, Caragianis S, Potter A. Autologous blood injection and wrist immobilization for chronic lateral epicondylitis. Adv Orthop 2012;2012:387829.

17. Thanasas C, Papadimitriou G, Charalambidis C, Paraskevopoulos I, Papanikolaoua A. Platelet-rich plasma versus autologous whole blood for the treatment of chronic lateral epicondylitis: a randomized controlled clinical trial. Am J Sports Med 2011;39:2130-4. [CrossRef] 